\title{
Post traumatic stress disorder symptoms in a psychiatric population not presenting with trauma: a preliminary study
}

\section{Mkize}

Department of Psychiatry, Nelson Mandela Faculty of Medicine, College of Health Sciences, University of KwaZulu-Natal, Durban, South Africa

\begin{abstract}
Objective: Post Traumatic Stress Disorder (PTSD) is a diagnostic category used to describe symptoms arising from emotionally traumatic experience(s). Research suggests that PTSD may be under- diagnosed when trauma is not the presenting problem or when not the focus of clinical intervention. There is a dearth of South African information on the prevalence of PTSD in a psychiatric population. The aim of this study was to determine the prevalence and comorbidity of PTSD in a psychiatric population, not presenting on the basis of trauma. Method: The study was cross sectional and conducted at a psychiatric outpatient clinic in the Durban Metropolitan area. The sample was obtained from patients seen at follow up over a period of twelve weeks. The researcher randomly selected prospective participants and at the end of their consultation the purpose of the study was explained and they were invited to participate. Demographic characteristics and diagnosis were recorded. Thereafter, the Zulu version of the Modified Posttraumatic Diagnostic Scale (MPDS) was administered and data collected. Results: The study demonstrated that $22 \%$ of subjects reported symptoms of PTSD where the primary presentation was not trauma related. Conclusion: A significant number of psychiatric patients presenting for non-trauma related psychopathology report symptoms of PTSD when specifically questioned. The findings suggest that such questioning may be overlooked when dealing with psychiatric patients who do not specifically present on the basis of trauma.
\end{abstract}

Key words: Stress-disorders; Post-traumatic; Mentally ill persons

Submitted: 12-05-2006

Accepted: 07-05-2007

\section{Introduction}

Post Traumatic Stress Disorder (PTSD) is an anxiety disorder currently defined by the coexistence of three clusters of symptoms, persisting for one month in survivors of a serious traumatic event .These clusters are:

- Intrusive cluster - Intrusions can take the form of repeated, unwanted and uncontrollable thoughts of the trauma, and can include nightmare and/or flashbacks.

- Avoidant cluster - These symptoms consist of the person's attempt to reduce exposure to people or places that may elicit memories of the event (or intrusive symptoms). This also involves symptoms such as social withdrawal, emotional numbing and a sense of loss of pleasure.

\section{Correspondence:}

Prof DL Mkize, Department of Psychiatry, Nelson Mandela

Faculty of Medicine, College of Health Sciences,

University of KwaZulu-Natal, Durban, South Africa

email: mkizedl@ukzn.ac.za
- Hyperarousal cluster - This refers to physiological signs of increased arousal, such as hypervigilance, or increased startle response..$^{1,2}$

Examples of the traumatic events which may result in PTSD are rape, hijack, armed robbery, serious car accident, kidnap, being diagnosed with a life threatening illness and natural disasters. ${ }^{1}$ While primary care physicians may identify symptoms of depression and anxiety, they may overlook the symptoms of PTSD more especially if trauma is not the presenting complaint. ${ }^{3}$

\section{Prevalence of PTSD}

Epidemiologic research has documented that most people experience a potentially traumatic event at least once in their lives. ${ }^{2}$ Estimates of the life-time prevalence of PTSD from surveys of the general population have ranged from $1 \%$ to $12.3 \%$. Other estimates suggest that the incidence and lifetime prevalence rates of PTSD in the general population 
are around 1\% - 9\%. These levels increase markedly for young adults living in inner cities (23\%) and for wounded combat veterans (20\%). In particularly violent areas, the incidence may be even higher. ${ }^{3}$ In South Africa, for example, it was found that of a politically displaced sample ( $\mathrm{n}=95)$ in KwaZulu-Natal in 1990, 87\% had symptoms which fulfilled the criteria for the diagnosis of PTSD. ${ }^{4}$ A further South African study of an urban Xhosa primary care population established a PTSD prevalence of $20 \% .{ }^{5}$ Within the South African context it appears that both setting and context influence prevalence rates.

\section{Co-morbidity}

In psychiatric epidemiology, comorbidity generally refers to the simultaneous occurrence of two or more mental disorders in one individual. Co-morbidity is common in PTSD, with a significant proportion of patients diagnosed as suffering from concurrent depression, anxiety disorders, cognitive impairment, and substance abuse. In a study of a community sample, Alexander, McFarlane and Papay ${ }^{6}$ demonstrated that only $28 \%$ of subjects who developed PTSD did not have a concurrent disorder. Both clinical and community studies have demonstrated that most people who have PTSD meet the criteria for at least one other psychiatric disorder as well. Data from the National Comorbidity Survey has been used to explore the issue of whether PTSD is primary or secondary in relation to co-morbid psychiatric diagnoses. Using temporal precedence as indicating the primary diagnosis, the suggestion is that PTSD is usually primary. ${ }^{2}$

\section{Rationale for the study}

PTSD may be underdiagnosed when trauma is not the presenting problem. It is important to understand that the patient often does not want to share the terrible experiences with the therapist. ${ }^{2}$ As there is a dearth of information on the prevalence of PTSD and PTSD as a comorbid disorder in South African psychiatric populations, the current study aimed to determine the prevalence and comorbidity of PTSD in a psychiatric population whose presentation for treatment was not trauma related. It was hypothesized that PTSD and comorbidity are prevalent in such a psychiatric population and often go undiagnosed.

\section{Methodology}

This was a cross sectional study undertaken at a psychiatric outpatient clinic in the Durban Metropolitan area. Over a period of twelve weeks patients seen at follow up by the researcher were randomly selected and invited to participate in the study at the end of the consultation for which the patients had presented for treatment. The purpose of the study was explained and permission to participate sought. Demographic characteristics, and diagnosis were recorded. Thereafter, the Zulu version of the Modified Posttraumatic Diagnostic Scale (MPDS) ${ }^{7}$ was administered and data collected.

\section{Results}

A total of 18 patients were interviewed. All patients were Africans; $24-67$ years (mean age $=42,5$ years); unemployed and unmarried. There were 10 males and 8 females. Most were illiterate; and none had tertiary education.

\section{Diagnoses}

None of the patients had a diagnosis of PTSD in the charts. The range of diagnoses was as follows:

$\begin{array}{ll}\text { Schizophrenia } & \mathrm{n}=8 \\ \text { Schizoaffective } & \mathrm{n}=3 \\ \text { Bipolar } & \mathrm{n}=2 \\ \text { Major Depression } & \mathrm{n}=1 \\ \text { Substance Induced Psychosis } & \mathrm{n}=2 \\ \text { Epileptic Psychosis } & \mathrm{n}=1 \\ \text { Mental Retardation } & \mathrm{n}=1\end{array}$

\section{Number of patients reporting a stressor}

Of the 18 patients interviewed, 8 (44\% of the sample) had experienced a serious traumatic stressor in the past year. Of these 8 patients, 4 reported symptoms suggesting a diagnosis of PTSD as per the MPDS score (Table I); a prevalence of $22 \%$.

\begin{tabular}{|l|c|c|c|c|c|c|c|c|}
\hline Table I & \multicolumn{7}{|c|}{ PATIENTS } \\
\hline MPDS SCORE (0-3 = NO PTSD, 4-5 = MOD, 6= SEVERE) \\
\hline SYMPTOMS & 1 & 2 & 3 & 4 & 5 & 6 & 7 & 8 \\
\hline Painful images & & & & $\times$ & & & $\times$ & \\
Distressing dreams & & & & $\times$ & $\times$ & & $\times$ & $\times$ \\
Reliving the stressor & & & & & $\times$ & $\times$ & $\times$ & \\
Psychological distress & $\times$ & & & $\times$ & $\times$ & $\times$ & & \\
Sleep problems & & & $\times$ & $\times$ & $\times$ & $\times$ & $\times$ & $\times$ \\
Easily startled & & & $\times$ & $\times$ & $\times$ & $\times$ & $\times$ & $\times$ \\
Avoidance & & & $\times$ & & $\times$ & & $\times$ & $\times$ \\
\hline TOTAL & 2 & 0 & 3 & 5 & 6 & 5 & 5 & 3 \\
\hline
\end{tabular}

The stressors reported were as follows:

- "My friend was stabbed to death in front of me. He died in my arms"

- "I was repairing a roof at home when I slipped and fell down, breaking my back I have frequent dreams of falling from heights"

- "Two people broke the door and entered our house. I pretended that I was asleep and could hear them discussing whether to kill me or not".

- I was sitting at home when two policemen arrived and forcefully arrested me. I had done no wrong. I spent two nights in prison and was released without any explanation. Whenever I see a police van I break into a sweat and I start shaking.

- I was in a taxi when I heard a loud bang. It had collided with a truck. There was blood and dead bodies all over. Ever since, I have not ridden a taxi".

- "Someone was shot at the taxi rank in front of me".

- "I took my child to hospital because of severe diarrhoea. He died in my arms while we were waiting in the queue to be seen by a doctor. I have bad dreams of my child crying out for help".

- This 25year old lady was gang raped and developed severe flashbacks and major depressive episode 


\section{Discussion}

This study showed a prevalence of PTSD symptoms in $22 \%$ of this population of psychiatric patients where the primary diagnosis was not PTSD and the reason for presentation was not trauma related. This finding is consistent with other South African data where a prevalence of $20 \%$ in an urban, Xhosa population in a primary care setting was established. ${ }^{5}$ The current study found comorbidity of PTSD with non-trauma related psychiatric diagnoses. This suggests that PTSD may well be overlooked when the presenting symptoms are not trauma related and if the PTSD symptoms are not specifically ascertained. A further finding of note was that symptoms which were most commonly reported were an increased startle response and sleep disturbances (Table I). Specifically that these were common to all for patients in whom a diagnosis of PTSD was suggested. A major limitation of the study is the sample size, which together with the demographic profile of the sample make it impossible to generalize these findings to the broader population of psychiatric patients. It does appear that further research utilizing a larger, more representative sample is warranted in order to meaningfully explore the issue of undiagnosed PTSD in psychiatric patients.

\section{Conclusion}

These findings are important in highlighting a potentially overlooked clinical problem which could have implications for the management of persons with psychiatric diagnoses. They emphasize the need to always ask and probe for the symptoms of PTSD so as to improve the diagnosis and management of patients who may have PTSD. Within the South African setting, this is especially relevant.

\section{References}

1. American Psychiatric Association. Diagnostic and Statistical Manual of Mental Disorders, Fourth Edition: APA, 1994.

2. Nutt D, Davidson JRT, Zohar J, eds. Post Traumatic Stress Disorder: Diagnosis, Management and Treatment. Martin Dumitz Ltd, 2000.

3. http://wWw. CSvr.org.za/papers/paptsd.htm

4. Michelson CL. Township violence, levels of distress and post traumatic stress disorder. In displacees from Natal. Psychology in Society 1994; 18: 47-55.

5. Carey PD, Stein DJ, Zungu-Dirwayi N, Seedat S. Trauma and post traumatic stress disorder in an urban Xhosa primary care population: prevalence, comorbidity and service use patterns. Journal of Nervous and Mental Disorders 2003 April; 191 (4):230236.

6. Alexander C, McFarlane MD, Papay P. Multiple diagnoses in PTSD in the victims of a natural disaster. Journal of Nervous and Mental Disorders 1992; 180 : 498 - 504.

7. Sheeran T, Zimmerman M. Screening for Post Traumatic Stress Disorder in a general psychiatric outpatient setting. Journal of Consulting and Clinical Psychology 2002 August; 70(4) : 961-966. 